\title{
On volume functions of special flow polytopes associated to the root system of type $A$
}

\author{
Takayuki Negishi Yuki Sugiyama \\ Department of Mathematics \\ Graduate School of Science and Engineering \\ Chuo University \\ Kasuga, Bunkyo-Ku, Tokyo \\ 112-8551 Japan \\ $\{$ n14009,y-sugi\}@gug.math. chuo-u.ac.jp
}

\author{
Tatsuru Takakura \\ Department of Mathematics \\ Chuo University \\ Kasuga, Bunkyo-Ku, Tokyo \\ 112-8551 Japan \\ takakura@math.chuo-u.ac.jp
}

Submitted: Oct 16, 2019; Accepted: Dec 11, 2020; Published: Dec 24, 2020

(C) The authors. Released under the CC BY-ND license (International 4.0).

\begin{abstract}
In this paper, we consider the volume of a special kind of flow polytope. We show that its volume satisfies a certain system of differential equations, and conversely, the solution of the system of differential equations is unique up to a constant multiple. In addition, we give an inductive formula for the volume with respect to the rank of the root system of type $A$.
\end{abstract}

Mathematics Subject Classifications: 52B20, 05A16

\section{Introduction}

The number of lattice points and the volume of a convex polytope are important and interesting objects and have been studied from various points of view (see, e.g., [4]). For example, the number of lattice points of a convex polytope associated to a root system is called the Kostant partition function, and it plays an important role in representation theory of Lie groups (see, e.g., [9]).

We consider a flow polytope associated to the root system of type $A$. As explained in $[2,3]$, the cone spanned by the positive roots is divided into several polyhedral cones called chambers, and the combinatorial property of a flow polytope depends on a chamber. Moreover, there is a specific chamber called the nice chamber, which plays a significant role in [11]. In this paper, we call a flow polytope for the nice chamber a special flow polytope. Also in $[2,3]$, a number of theoretical results related to the Kostant partition function and the volume function of a flow polytope can be found. In particular, it is shown that these functions for the nice chamber are written as iterated residues ([3, Lemma 21]). We also refer to [1] for similar formulas for other chambers in more general settings. Moreover, we 
mention that a generalization of the Lidskii formula is shown in [3, Theorem 38], there is a geometric proof of the Lidskii formula in [12], and combinatorial applications of this formula are given in $[5,7]$.

The purpose of this paper is to characterize the volume function of a flow polytope for the nice chamber in terms of a system of differential equations, based on a result in [3]. In order to state the main results, we give some notation. Let $e_{1}, \ldots, e_{r+1}$ be the standard basis of $\mathbb{R}^{r+1}$ and let

$$
A_{r}^{+}=\left\{e_{i}-e_{j} \mid 1 \leqslant i<j \leqslant r+1\right\}
$$

be the positive root system of type $A$ with rank $r$. We assign a positive integer $m_{i, j}$ to each $i$ and $j$ with $1 \leqslant i<j \leqslant r+1$. Let us set $m=\left(m_{i, j}\right)$ and $M=\sum_{1 \leqslant i<j \leqslant r+1} m_{i, j}$. For $a=a_{1} e_{1}+\cdots+a_{r} e_{r}-\left(a_{1}+\cdots+a_{r}\right) e_{r+1} \in \mathbb{R}^{r+1}$, where $a_{i} \in \mathbb{R}_{\geqslant 0}(i=1, \ldots, r)$, the following polytope $P_{A_{r}^{+}, m}(a)$ is called the flow polytope associated to the root system of type $A$ :

$$
P_{A_{r}^{+}, m}(a)=\left\{\begin{array}{l|l}
\left(y_{i, j, k}\right) \in \mathbb{R}^{M} & \begin{array}{l}
1 \leqslant i<j \leqslant r+1,1 \leqslant k \leqslant m_{i, j}, y_{i, j, k} \geqslant 0, \\
\sum_{1 \leqslant i<j \leqslant r+1} \sum_{1 \leqslant k \leqslant m_{i, j}} y_{i, j, k}\left(e_{i}-e_{j}\right)=a
\end{array}
\end{array}\right\} .
$$

Note that the flow polytopes in [3] include the case that some of $m_{i, j}$ 's are zero, whereas we exclude such cases in this paper. We denote the volume of $P_{A_{r}^{+}, m}(a)$ by $v_{A_{r}^{+}, m}(a)$.

The open set

$$
\mathfrak{c}_{\text {nice }}:=\left\{a=a_{1} e_{1}+\cdots+a_{r} e_{r}-\left(a_{1}+\cdots+a_{r}\right) e_{r+1} \in \mathbb{R}^{r+1} \mid a_{i}>0, i=1, \ldots, r\right\}
$$

in $\mathbb{R}^{r+1}$ is called the nice chamber. We are interested in the volume $v_{A_{r}^{+}, m}(a)$ when $a$ is in the closure of the nice chamber, and then it is written by $v_{A_{r}^{+}, m, \mathfrak{c}_{\text {nice }}}$. It is a homogeneous polynomial of degree $M-r$. The first result of this paper is the following.

Theorem 1. Let $a=\sum_{i=1}^{r} a_{i}\left(e_{i}-e_{r+1}\right) \in \overline{\mathfrak{c}_{\text {nice }}}$, and let $v_{A_{r}^{+}, m, \mathfrak{c}_{\text {nice }}}(a)$ be the volume of $P_{A_{r}^{+}, m}(a)$. Then $v=v_{A_{r}^{+}, m, \mathfrak{c}_{\text {nice }}}(a)$ satisfies the system of differential equations as follows:

$$
\left\{\begin{array}{l}
\partial_{r}^{m_{r, r+1}} v=0 \\
\left(\partial_{r-1}-\partial_{r}\right)^{m_{r-1, r}} \partial_{r-1}^{m_{r-1, r+1}} v=0 \\
\quad \vdots \\
\left(\partial_{1}-\partial_{2}\right)^{m_{1,2}}\left(\partial_{1}-\partial_{3}\right)^{m_{1,3}} \cdots\left(\partial_{1}-\partial_{r}\right)^{m_{1, r}} \partial_{1}^{m_{1, r+1}} v=0
\end{array}\right.
$$

where $\partial_{i}=\frac{\partial}{\partial a_{i}}$ for $i=1, \ldots, r$. Conversely, the polynomial $v=v(a)$ of degree $M-r$ satisfying the above equations is equal to a constant multiple of $v_{A_{r}^{+}, m, \mathfrak{c}_{\text {nice }}}(a)$.

We remark that it is known that the volume function $v_{A_{r}^{+}, m}(a)$ of $P_{A_{r}^{+}, m}(a)$, as a distribution on $\mathbb{R}^{r}$, satisfies the differential equation

$$
L v_{A_{r}^{+}, m}(a)=\delta(a)
$$

in general, where $L=\prod_{i<j}\left(\partial_{i}-\partial_{j}\right)^{m_{i, j}}$ and $\delta(a)$ is the Dirac delta function on $\mathbb{R}^{r}([8$, 11]). Note that $\partial_{r+1}$ in the definition of $L$ is supposed to be zero. The above theorem 
characterizes the function $v_{A_{r}^{+}, m, \mathfrak{c}_{\text {nice }}}(a)$ on $\overline{\mathfrak{c}_{\text {nice }}}$ more explicitly. It might be interesting to see what kind of properties of the volume can be derived from Theorem 1.

In addition, in Theorem 20, we show the volume $v_{A_{r}^{+}, m, \mathbf{c}_{\text {nice }}}(a)$ is written by a linear combination of $v_{A_{r-1}^{+}, m^{\prime}, \mathfrak{c}_{\text {nice }}^{\prime}}\left(a^{\prime}\right)$ and its partial derivatives, where $m^{\prime}=\left(m_{i, j}\right)_{2 \leqslant i<j \leqslant r+1}, \mathfrak{c}_{\text {nice }}^{\prime}$ is the nice chamber of $A_{r-1}^{+}$, and $a^{\prime}=\sum_{i=2}^{r} a_{i}\left(e_{i}-e_{r+1}\right) \in \overline{\mathfrak{c}_{\text {nice }}^{\prime}}$. It might be interesting to ask whether there is a relaton between this theorem and the inductive formulas of Schmidt-Bincer [13, (4.1), (4.24)].

This paper is organized as follows. In Section 2, we recall the iterated residue, the Jeffrey-Kirwan residue, and the nice chamber based on [2], [3], [6] and [10]. Also, we give some examples of $P_{A_{r}^{+}, m}(a)$ and the calculations of the volume $v_{A_{r}^{+}, m, \mathfrak{c}_{\text {nice }}}(a)$. In Section 3 , we prove the main theorems.

\section{Preliminaries}

In this section, we set up the tools to prove the main theorems based on [2], [3], [6] and [10].

\subsection{Flow polytopes and its volumes}

Let $e_{1}, \ldots, e_{r+1}$ be the standard basis of $\mathbb{R}^{r+1}$, and let

$$
V=\left\{a=\sum_{i=1}^{r+1} a_{i} e_{i} \in \mathbb{R}^{r+1} \mid \sum_{i=1}^{r+1} a_{i}=0\right\} .
$$

We consider the positive root system of type $A$ with rank $r$ as follows:

$$
A_{r}^{+}=\left\{e_{i}-e_{j} \mid 1 \leqslant i<j \leqslant r+1\right\} \text {. }
$$

Let $C\left(A_{r}^{+}\right)$be the convex cone generated by $A_{r}^{+}$:

$$
C\left(A_{r}^{+}\right)=\left\{a=a_{1} e_{1}+\cdots+a_{r} e_{r}-\left(a_{1}+\cdots+a_{r}\right) e_{r+1} \mid a_{1}, \ldots, a_{r} \in \mathbb{R}_{\geqslant 0}\right\} .
$$

We assign a positive integer $m_{i, j}$ to each $i$ and $j$ with $1 \leqslant i<j \leqslant r+1$, and it is called a multiplicity. Let us set $m=\left(m_{i, j}\right)$ and $M=\sum_{1 \leqslant i<j \leqslant r+1} m_{i, j}$.

Definition 2. Let $a=a_{1} e_{1}+\cdots+a_{r} e_{r}-\left(a_{1}+\cdots+a_{r}\right) e_{r+1} \in C\left(A_{r}^{+}\right)$. We consider the following polytope:

$$
P_{A_{r}^{+}, m}(a)=\left\{\begin{array}{l|l}
\left(y_{i, j, k}\right) \in \mathbb{R}^{M} & \begin{array}{l}
1 \leqslant i<j \leqslant r+1,1 \leqslant k \leqslant m_{i, j}, y_{i, j, k} \geqslant 0, \\
\sum_{1 \leqslant i<j \leqslant r+1} \sum_{1 \leqslant k \leqslant m_{i, j}} y_{i, j, k}\left(e_{i}-e_{j}\right)=a
\end{array}
\end{array}\right\},
$$

which is called the flow polytope associated to the root system of type $A$.

Remark 3. The flow polytopes in [3] include the case that $m_{i, j}=0$ for some $i$ and $j$. 
The elements of $A_{r}^{+}$generate a lattice $V_{\mathbb{Z}}$ in $V$. The lattice $V_{\mathbb{Z}}$ determines a measure $d a$ on $V$.

Let $d u$ be the Lebesgue measure on $\mathbb{R}^{M}$. Let $\left[\alpha_{1}, \ldots, \alpha_{M}\right]$ be a sequence of elements of $A_{r}^{+}$with multiplicity $m_{i, j}$, and let $\varphi$ be the surjective linear map from $\mathbb{R}^{M}$ to $V$ defined by $\varphi\left(e_{k}\right)=\alpha_{k}$. The vector space $\operatorname{ker}(\varphi)=\varphi^{-1}(0)$ is of dimension $d=M-r$ and it is equipped with the quotient Lebesgue measure $d u / d a$. For $a \in V$, the affine space $\varphi^{-1}(a)$ is parallel to $\operatorname{ker}(\varphi)$, and thus also equipped with the Lebesgue measure $d u / d a$. Volumes of subsets of $\varphi^{-1}(a)$ are computed for this measure. In particular, we can consider the volume $v_{A_{r}^{+}, m}(a)$ of the polytope $P_{A_{r}^{+}, m}(a)$.

\subsection{Total residue and iterated residue}

Let $A_{r}=A_{r}^{+} \cup\left(-A_{r}^{+}\right)$, and let $U$ be the dual vector space of $V$. We denote by $R_{A_{r}}$ the ring of rational functions $f\left(x_{1}, \ldots, x_{r}\right)$ on the complexification $U_{\mathbb{C}}$ of $U$ with poles on the hyperplanes $x_{i}-x_{j}=0(1 \leqslant i<j \leqslant r+1)$ or $x_{i}=0(1 \leqslant i \leqslant r)$. A subset $\sigma$ of $A_{r}$ is called a basis of $A_{r}$ if the elements $\alpha \in \sigma$ form a basis of $V$. In this case, we set

$$
f_{\sigma}(x):=\frac{1}{\prod_{\alpha \in \sigma} \alpha(x)}
$$

and call such a element a simple fraction. We denote by $S_{A_{r}}$ the linear subspace of $R_{A_{r}}$ spanned by simple fractions. The space $U$ acts on $R_{A_{r}}$ by differentiation: $(\partial(u) f)(x)=$ $\left.\left(\frac{d}{d \varepsilon}\right) f(x+\varepsilon u)\right|_{\varepsilon=0}$. We denote by $\partial(U) R_{A_{r}}$ the space spanned by derivatives of functions in $R_{A_{r}}$. It is shown in [6, Proposition 7] that $R_{A_{r}}=\partial(U) R_{A_{r}} \oplus S_{A_{r}}$. The projection map Tres $_{A_{r}}: R_{A_{r}} \rightarrow S_{A_{r}}$ with respect to this decomposition is called the total residue map.

We extend the definition of the total residue to the space $\hat{R}_{A_{r}}$ consisting of functions $P / Q$ where $Q$ is a finite product of powers of the linear forms $\alpha \in A_{r}$ and $P=\sum_{k=0}^{\infty} P_{k}$ is a formal power series with $P_{k}$ of degree $k$. As the total residue vanishes outside the homogeneous component of degree $-r$ of $A_{r}$, we can define $\operatorname{Tres}_{A_{r}}(P / Q)=\operatorname{Tres}_{A_{r}}\left(P_{q-r} / Q\right)$, where $q$ is degree of $Q$. For $a \in V$ and multiplicities $m=\left(m_{i, j}\right) \in\left(\mathbb{Z}_{\geqslant 0}\right)^{M}$ of elements of $A_{r}^{+}$, the function

$$
F:=\frac{e^{a_{1} x_{1}+\cdots+a_{r} x_{r}}}{\prod_{i=1}^{r} x_{i}^{m_{i, r+1}} \prod_{1 \leqslant i<j \leqslant r}\left(x_{i}-x_{j}\right)^{m_{i, j}}}
$$

is in $\hat{R}_{A_{r}}$. We define $J_{A_{r}^{+}, m}(a) \in S_{A_{r}}$ by

$$
J_{A_{r}^{+}, m}(a)=\operatorname{Tres}_{A_{r}} F .
$$

Next, we describe the iterated residue.

Definition 4. For $f \in R_{A_{r}}$, we define the iterated residue by

$$
\operatorname{Ires}_{x=0} f=\operatorname{Res}_{x_{1}=0} \operatorname{Res}_{x_{2}=0} \cdots \operatorname{Res}_{x_{r}=0} f\left(x_{1}, \ldots, x_{r}\right) .
$$

Since the iterated residue $\operatorname{Ires}_{x=0} f$ vanishes on the space $\partial(U) R_{A_{r}}$ as in [3], we have

$$
\operatorname{Ires}_{x=0} J_{A_{r}^{+}, m}(a)=\operatorname{Ires}_{x=0} F .
$$




\subsection{Chambers and Jeffrey-Kirwan residue}

Definition 5. Let $C(\nu)$ be the closed cone generated by $\nu$ for any subset $\nu$ of $A_{r}^{+}$and let $C\left(A_{r}^{+}\right)_{\text {sing }}$ be the union of the cones $C(\nu)$ where $\nu$ is any subset of $A_{r}^{+}$of cardinal strictly less than $r=\operatorname{dim} V$. By definition, the set $C\left(A_{r}^{+}\right)_{\text {reg }}$ of $A_{r}^{+}$-regular elements is the complement of $C\left(A_{r}^{+}\right)_{\text {sing. }}$. A connected component of $C\left(A_{r}^{+}\right)_{\text {reg }}$ is called a chamber.

The Jeffrey-Kirwan residue [10] associated to a chamber $\mathfrak{c}$ of $C\left(A_{r}^{+}\right)$is a linear form $f \mapsto\langle\langle\mathfrak{c}, f\rangle\rangle$ on the vector space $S_{A_{r}}$ of simple fractions. Any function $f$ in $S_{A_{r}}$ can be written as a linear combination of functions $f_{\sigma}$, with a basis $\sigma$ of $A_{r}$ contained in $A_{r}^{+}$. To determine the linear map $f \mapsto\langle\langle\mathfrak{c}, f\rangle\rangle$, it is enough to determine it on this set of functions $f_{\sigma}$. So we assume that $\sigma$ is a basis of $A_{r}$ contained in $A_{r}^{+}$.

Definition 6. For a chamber $\mathfrak{c}$ and $f_{\sigma} \in S_{A_{r}}$, we define the Jeffrey-Kirwan residue $\left\langle\left\langle\mathfrak{c}, f_{\sigma}\right\rangle\right\rangle$ associated to a chamber $\mathfrak{c}$ as follows:

- If $\mathfrak{c} \subset C(\sigma)$, then $\left\langle\left\langle\mathfrak{c}, f_{\sigma}\right\rangle\right\rangle=1$.

- If $\mathfrak{c} \cap C(\sigma)=\emptyset$, then $\left\langle\left\langle\mathfrak{c}, f_{\sigma}\right\rangle\right\rangle=0$,

where $C(\sigma)$ is the convex cone generated by $\sigma$.

Remark 7. More generally, as in [3, Definition 11], the Jeffrey-Kirwan residue $\left\langle\left\langle\mathfrak{c}, f_{\sigma}\right\rangle\right\rangle$ is defined to be $\frac{1}{\operatorname{vol}(\sigma)}$ if $\mathfrak{c} \subset C(\sigma)$, where $\operatorname{vol}(\sigma)$ is the volume of the parallelepiped $\oplus_{\alpha \in \sigma}[0,1] \alpha$, relative to our Lebesgue measure $d a$. In our case, the volume $\operatorname{vol}(\sigma)$ is equal to 1 since $A_{r}$ is unimodular.

The volume $v_{A_{r}^{+}, m}(a)$ of the flow polytope $P_{A_{r}^{+}, m}(a)$ is written by the function $J_{A_{r}^{+}, m}(a)$ and the Jeffrey-Kirwan residue in the following.

Theorem 8 (Baldoni-Vergne [3]). Let $\mathfrak{c}$ be a chamber of $C\left(A_{r}^{+}\right)$. Then, for $a \in \overline{\mathfrak{c}}$, the volume $v_{A_{r}^{+}, m}(a)$ of $P_{A_{r}^{+}, m}(a)$ is given by

$$
v_{A_{r}^{+}, m}(a)=\left\langle\left\langle\mathfrak{c}, J_{A_{r}^{+}, m}(a)\right\rangle\right\rangle .
$$

We denote by $v_{A_{r}^{+}, m, \mathfrak{c}}(a)$ the polynomial function of $a$ coinciding with $v_{A_{r}^{+}, m}(a)$ when $a \in \overline{\mathfrak{c}}$. It is a homogeneous polynomial of degree $M-r$.

\subsection{Nice chamber}

Definition 9. The open subset $\mathfrak{c}_{\text {nice }}$ of $C\left(A_{r}^{+}\right)$is defined by

$$
\mathfrak{c}_{\text {nice }}=\left\{a \in C\left(A_{r}^{+}\right) \mid a_{i}>0(i=1, \ldots, r)\right\} .
$$

The set $\mathfrak{c}_{\text {nice }}$ is in fact a chamber for the root system $A_{r}^{+}([3])$. The chamber $\mathfrak{c}_{\text {nice }}$ is called the nice chamber.

Lemma 10 (Baldoni-Vergne [3]). For the nice chamber $\mathfrak{c}_{\text {nice }}$ of $A_{r}^{+}$and $f \in S_{A_{r}}$, we have

$$
\left\langle\left\langle\mathfrak{c}_{\text {nice }}, f\right\rangle\right\rangle=\operatorname{Ires}_{x=0} f .
$$


From Theorem 8, Lemma 10 and (1), we have the following corollary.

Corollary 11 (Lidskii formula [3]). Let $a \in \overline{\mathfrak{c}_{\text {nice }}}$. Then the volume function $v_{A_{r}^{+}, m, \mathfrak{c}_{\text {nice }}}(a)$ is given by

$$
v_{A_{r}^{+}, m, \mathfrak{c}_{\text {nice }}}(a)=\operatorname{Ires}_{x=0} F .
$$

\subsection{Examples}

In this subsection, we give some examples of the flow polytopes for $A_{1}, A_{2}$, and $A_{3}$, and calculate their volumes.

Example 12. When $r=1$, the nice chamber of $A_{1}^{+}$is $\mathfrak{c}_{\text {nice }}=\left\{a=a_{1}\left(e_{1}-e_{2}\right) \mid a_{1}>0\right\}$. For $a=a_{1}\left(e_{1}-e_{2}\right) \in \overline{\mathfrak{c}_{\text {nice }}}$,

$$
P_{A_{1}^{+}, m}(a)=\left\{\left(y_{i, j, k}\right) \in \mathbb{R}^{m_{1,2}} \mid y_{i, j, k} \geqslant 0, y_{1,2,1}+y_{1,2,2}+\cdots+y_{1,2, m_{1,2}}=a_{1}\right\} .
$$

From Corollary 11, we have

$$
v_{A_{1}^{+}, m, \mathfrak{c}_{\text {nice }}}(a)=\operatorname{Res}_{x_{1}=0}\left(\frac{e^{a_{1} x_{1}}}{x_{1}^{m_{1,2}}}\right)=\frac{1}{\left(m_{1,2}-1\right) !} a_{1}^{m_{1,2}-1} .
$$

Example 13. When $r=2$, there are two chambers $\mathfrak{c}_{1}, \mathfrak{c}_{2}$ of $A_{2}^{+}$as below, and the nice chamber $\mathfrak{c}_{\text {nice }}$ of $A_{2}^{+}$is $\mathfrak{c}_{1}$.

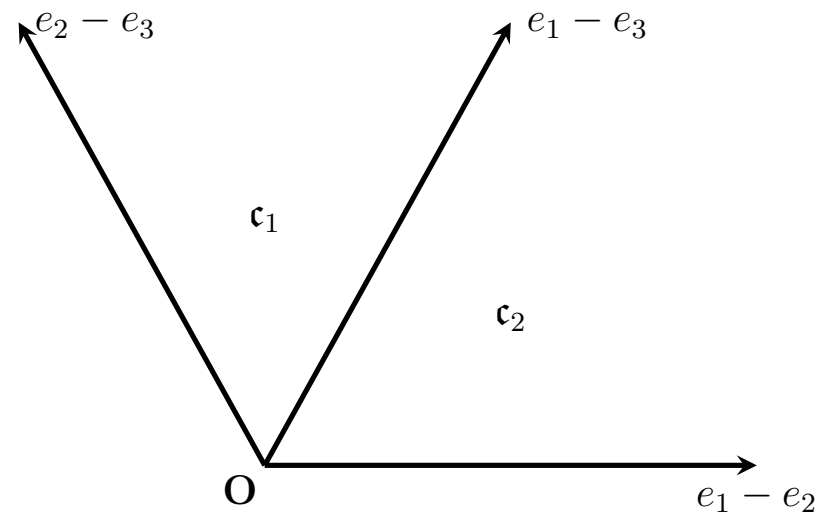

Figure 1 : The chamber of $A_{2}^{+}$.

For example, we set $m_{1,2}=n\left(n \in \mathbb{Z}_{>0}\right), m_{1,3}=1$, and $m_{2,3}=1$. For $a=a_{1} e_{1}+$ $a_{2} e_{2}-\left(a_{1}+a_{2}\right) e_{3} \in \overline{\mathfrak{c}_{\text {nice }}}$,

$$
P_{A_{2}^{+}, m}(a)=\left\{\begin{array}{l|l}
\left(y_{i, j, k}\right) \in \mathbb{R}^{n+2} & \begin{array}{l}
y_{i, j, k} \geqslant 0 \\
y_{1,2,1}+y_{1,2,2}+\cdots+y_{1,2, n}+y_{1,3,1}=a_{1} \\
-y_{1,2,1}-y_{1,2,2}-\cdots-y_{1,2, n}+y_{2,3,1}=a_{2}
\end{array}
\end{array}\right\} .
$$

From Corollary 11, we have

$$
v_{A_{2}^{+}, m, \mathfrak{c}_{\text {nice }}}(a)=\operatorname{Ires}_{x=0}\left(\frac{e^{a_{1} x_{1}+a_{2} x_{2}}}{x_{1} x_{2}\left(x_{1}-x_{2}\right)^{n}}\right)=\operatorname{Res}_{x_{1}=0} \operatorname{Res}_{x_{2}=0}\left(\frac{e^{a_{1} x_{1}+a_{2} x_{2}}}{x_{1} x_{2}\left(x_{1}-x_{2}\right)^{n}}\right)=\frac{1}{n !} a_{1}^{n} .
$$


Example 14. When $r=3$, there are seven chambers of $A_{3}^{+}$as below ([1]), and the nice chamber $\mathfrak{c}_{\text {nice }}$ of $A_{3}^{+}$is $\mathfrak{c}_{1}$.

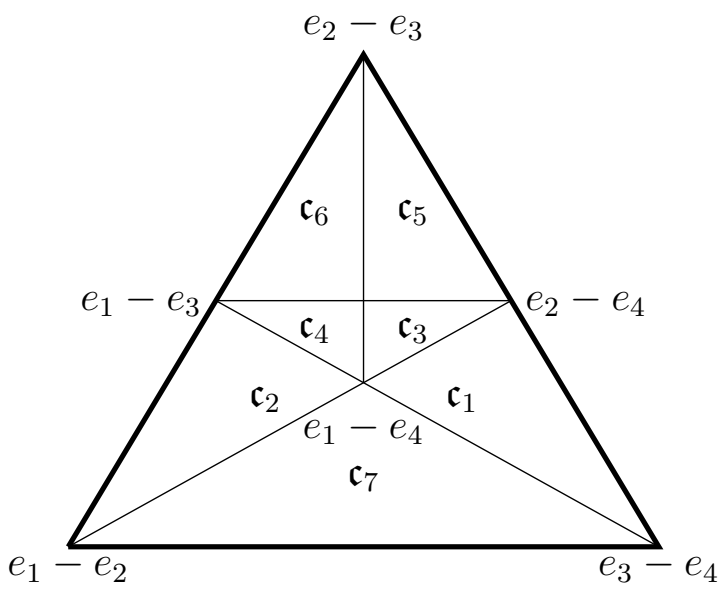

Figure 2 : The chamber of $A_{3}^{+}$.

For example, we set $m_{1,2}=1, m_{1,3}=1, m_{1,4}=2, m_{2,3}=1, m_{2,4}=2$, and $m_{3,4}=2$. For $a=\sum_{i=1}^{3} a_{i}\left(e_{i}-e_{4}\right) \in \overline{\mathfrak{c}_{\text {nice }}}$,

$$
P_{A_{3}^{+}, m}(a)=\left\{\begin{array}{l|l}
\left(y_{i, j, k}\right) \in \mathbb{R}^{9} & \begin{array}{l}
y_{i, j, k} \geqslant 0 \\
y_{1,2,1}+y_{1,3,1}+y_{1,4,1}+y_{1,4,2}=a_{1} \\
-y_{1,2,1}+y_{2,3,1}+y_{2,4,1}+y_{2,4,2}=a_{2} \\
-y_{1,3,1}-y_{2,3,1}+y_{3,4,1}+y_{3,4,2}=a_{3}
\end{array}
\end{array}\right\} .
$$

From Corollary 11, we have

$$
\begin{aligned}
v_{A_{3}^{+}, m, \mathfrak{c}_{\text {nice }}}(a) & =\operatorname{Ires}_{x=0}\left(\frac{e^{a_{1} x_{1}+a_{2} x_{2}+a_{3} x_{3}}}{x_{1}^{2} x_{2}^{2} x_{3}^{2}\left(x_{1}-x_{2}\right)\left(x_{1}-x_{3}\right)\left(x_{2}-x_{3}\right)}\right) \\
& =\frac{1}{360} a_{1}^{3}\left(a_{1}^{3}+6 a_{1}^{2} a_{2}+3 a_{1}^{2} a_{3}+15 a_{1} a_{2}^{2}+15 a_{1} a_{2} a_{3}+10 a_{2}^{3}+30 a_{2}^{2} a_{3}\right) .
\end{aligned}
$$

\section{Main theorems}

In this section, we prove the main theorems of this paper. Let $\mathfrak{c}_{\text {nice }}$ be the nice chamber of $A_{r}^{+}$and let $a=\sum_{i=1}^{r} a_{i}\left(e_{i}-e_{r+1}\right) \in \overline{\mathfrak{c}_{\text {nice }}}$.

Theorem 15. For $a \in \overline{\mathfrak{c}_{\text {nice }}}$, let $P_{A_{r}^{+}, m}(a)$ be the flow polytope as in Definition 2 and let $v_{A_{r}^{+}, m, \mathfrak{c}_{\text {nice }}}(a)$ be the volume of $P_{A_{r}^{+}, m}(a)$. Then $v=v_{A_{r}^{+}, m, \mathfrak{c}_{\text {nice }}}(a)$ satisfies the system of differential equations as follows:

$$
\left\{\begin{array}{l}
\partial_{r}^{m_{r, r+1}} v=0 \\
\left(\partial_{r-1}-\partial_{r}\right)^{m_{r-1, r}} \partial_{r-1}^{m_{r-1, r+1}} v=0 \\
\quad \vdots \\
\left(\partial_{1}-\partial_{2}\right)^{m_{1,2}}\left(\partial_{1}-\partial_{3}\right)^{m_{1,3}} \cdots\left(\partial_{1}-\partial_{r}\right)^{m_{1, r}} \partial_{1}^{m_{1, r+1}} v=0
\end{array}\right.
$$


where $\partial_{i}=\frac{\partial}{\partial a_{i}}$ for $i=1, \ldots, r$.

Proof. We will prove the first two relations. Let $F=\frac{e^{a_{1} x_{1}+\cdots+a_{r} x_{r}}}{\prod_{i=1}^{r} x_{i}^{m_{i, r+1}} \prod_{1 \leqslant i<j \leqslant r}\left(x_{i}-x_{j}\right)^{m_{i, j}}}$. It is easy to see that

$$
P\left(\partial_{1}, \ldots, \partial_{r}\right)\left(\operatorname{Ires}_{x=0} F\right)=\operatorname{Ires}_{x=0}\left(P\left(\partial_{1}, \ldots, \partial_{r}\right) F\right)=\operatorname{Ires}_{x=0}\left(P\left(x_{1}, \ldots, x_{r}\right) F\right),
$$

where $P$ is a polynomial. Since $\frac{e^{a_{1} x_{1}+\cdots+a_{k} x_{k}}}{\prod_{i=1}^{k-1} x_{i}^{m_{i}, r+1} \prod_{1 \leqslant i<j \leqslant k}\left(x_{i}-x_{j}\right)^{m_{i, j}}}$ is holomorphic at $x_{k}=0$,

$$
\operatorname{Res}_{x_{k}=0}\left(\frac{e^{a_{1} x_{1}+\cdots+a_{k} x_{k}}}{\prod_{i=1}^{k-1} x_{i}^{m_{i, r+1}} \prod_{1 \leqslant i<j \leqslant k}\left(x_{i}-x_{j}\right)^{m_{i, j}}}\right)=0
$$

for $k=1, \ldots, r$. Therefore, from Corollary 11, (3) and (4), we obtain

$$
\begin{aligned}
\partial_{r}^{m_{r, r+1}} v & =\partial_{r}^{m_{r, r+1}} \operatorname{Ires}_{x=0} F=\operatorname{Ires}_{x=0} \partial_{r}^{m_{r, r+1}} F \\
& =\operatorname{Ires}_{x=0}\left(\frac{e^{a_{1} x_{1}+\cdots+a_{r} x_{r}}}{\prod_{i=1}^{r-1} x_{i}^{m_{i, r+1}} \prod_{1 \leqslant i<j \leqslant r}\left(x_{i}-x_{j}\right)^{m_{i, j}}}\right)=0,
\end{aligned}
$$

and

$$
\begin{aligned}
& \left(\partial_{r-1}-\partial_{r}\right)^{m_{r-1, r}} \partial_{r-1}^{m_{r-1, r+1}} v \\
& =\operatorname{Ires}_{x=0}\left(\partial_{r-1}-\partial_{r}\right)^{m_{r-1, r}} \partial_{r-1}^{m_{r-1, r+1}} F \\
& =\operatorname{Ires}_{x=0}\left(\partial_{r-1}-\partial_{r}\right)^{m_{r-1, r}}\left(\frac{e^{a_{1} x_{1}+\cdots+a_{r} x_{r}}}{x_{r}^{m_{r, r+1}} \prod_{i=1}^{r-2} x_{i}^{m_{i, r+1}} \prod_{1 \leqslant i<j \leqslant r}\left(x_{i}-x_{j}\right)^{m_{i, j}}}\right) \\
& =\operatorname{Ires}_{x=0}\left(\frac{e^{a_{1} x_{1}+\cdots+a_{r} x_{r}}}{x_{r}^{m_{r, r+1}} \prod_{i=1}^{r-2} x_{i}^{m_{i, r+1}} \prod_{1 \leqslant i<j \leqslant r,(i, j) \neq(r-1, r)}\left(x_{i}-x_{j}\right)^{m_{i, j}}}\right) \\
& =\operatorname{Res}_{x_{1}=0} \cdots\left(\operatorname{Res}_{x_{r-1}=0}\left(\frac{e^{a_{1} x_{1}+\cdots+a_{r-1} x_{r-1}}}{\prod_{i=1}^{r-2} x_{i}^{m_{i, r+1}} \prod_{1 \leqslant i<j \leqslant r-1}\left(x_{i}-x_{j}\right)^{m_{i, j}}}\right)\right)=0 . \\
& \left.\left.\times \operatorname{Res}_{x_{r}=0}\left(\frac{e^{a_{r} x_{r}}}{x_{r}^{m_{r, r+1}} \prod_{i=1}^{r-2}\left(x_{i}-x_{r}\right)^{m_{i, r}}}\right)\right)\right)=0
\end{aligned}
$$

Similarly, we can verify the remaining expressions.

Remark 16. In general, it is known that the volume function $v_{A_{r}^{+}, m}(a)$ of $P_{A_{r}^{+}, m}(a)$, as a distribution on $V$, satisfies the differential equation

$$
L v_{A_{r}^{+}, m}(a)=\delta(a),
$$

where $L=\prod_{i<j}\left(\partial_{i}-\partial_{j}\right)^{m_{i, j}}$ and $\delta(a)$ is the Dirac delta function on $V([8,11])$. Note that $\partial_{r+1}$ in the definition of $L$ is supposed to be zero. Theorem 15 above, together with Proposition 17 and Theorem 18 as below, characterizes the function $v_{A_{r}^{+}, m, \mathfrak{c}_{\text {nice }}}(a)$ on $\overline{\mathfrak{c}_{\text {nice }}}$ more explicitly. 
Let $M_{\ell}=\sum_{i=\ell+1}^{r+1} m_{\ell, i}$ for $\ell=1, \ldots, r$. Then we have the following proposition.

Proposition 17. The coefficient of $a_{1}^{M_{1}-1} a_{2}^{M_{2}-1} \cdots a_{r-1}^{M_{r-1}-1} a_{r}^{M_{r}-1}$ in the volume function $v_{A_{r}^{+}, m}(a)$ is given by

$$
\frac{1}{\left(M_{1}-1\right) !\left(M_{2}-1\right) ! \cdots\left(M_{r-1}-1\right) !\left(M_{r}-1\right) !} .
$$

Proof. From the Lidskii formula in Corollary 11, we have

$$
v_{A_{r}^{+}, m, \mathfrak{c}_{\text {nice }}}(a)=\sum_{|i|=\ell-r} \frac{a_{1}^{i_{1}}}{i_{1} !} \cdots \frac{a_{r}^{i_{r}}}{i_{r} !} \operatorname{Ires}_{x=0}\left(\frac{x_{1}^{i_{1}} \cdots x_{r}^{i_{r}}}{\prod_{i=1}^{r} x_{i}^{m_{i, r+1}} \prod_{1 \leqslant i<j \leqslant r}\left(x_{i}-x_{j}\right)^{m_{i, j}}}\right),
$$

where $|i|=i_{1}+\cdots+i_{r}$. When $i_{\ell}=M_{\ell}-1$ for $\ell=1, \ldots, r$,

$$
\begin{aligned}
& \operatorname{Ires}_{x=0}\left(\frac{x_{1}^{M_{1}-1} \cdots x_{r}^{M_{r}-1}}{\prod_{i=1}^{r} x_{i}^{m_{i, r+1}} \prod_{1 \leqslant i<j \leqslant r}\left(x_{i}-x_{j}\right)^{m_{i, j}}}\right) \\
& =\operatorname{Res}_{x_{1}=0} \cdots \operatorname{Res}_{x_{r-1}=0} \operatorname{Res}_{x_{r}=0}\left(\frac{x_{1}^{\left(\sum_{i=2}^{r} m_{1, i}\right)-1} \cdots x_{r-1}^{m_{r-1, r}-1}}{x_{r} \prod_{1 \leqslant i<j \leqslant r}\left(x_{i}-x_{j}\right)^{m_{i, j}}}\right) \\
& =\operatorname{Res}_{x_{1}=0} \cdots \operatorname{Res}_{x_{r-1}=0}\left(\frac{x_{1}^{\left(\sum_{i=2}^{r-1} m_{1, i}\right)-1} \cdots x_{r-2}^{m_{r-2, r-1}-1}}{x_{r-1} \prod_{1 \leqslant i<j \leqslant r-1}\left(x_{i}-x_{j}\right)^{m_{i, j}}}\right) \\
& =\operatorname{Res}_{x_{1}=0} \frac{1}{x_{1}}=1 .
\end{aligned}
$$

Thus we obtain the proposition.

Theorem 18. Let $\phi_{r}=\phi\left(a_{1}, \ldots, a_{r}\right)$ be a homogeneous polynomial of $a_{1}, \ldots, a_{r}$ with degree $d$ and let $M=\sum_{1 \leqslant i<j \leqslant r+1} m_{i, j}$. Suppose $\phi_{r}$ satisfies the system of differential equations as follows:

$$
\left\{\begin{array}{l}
\partial_{r}^{m_{r, r+1}} \phi_{r}=0 \\
\left(\partial_{r-1}-\partial_{r}\right)^{m_{r-1, r}} \partial_{r-1}^{m_{r-1, r+1}} \phi_{r}=0 \\
\quad \vdots \\
\left(\partial_{1}-\partial_{2}\right)^{m_{1,2}}\left(\partial_{1}-\partial_{3}\right)^{m_{1,3}} \cdots\left(\partial_{1}-\partial_{r}\right)^{m_{1, r}} \partial_{1}^{m_{1, r+1}} \phi_{r}=0 .
\end{array}\right.
$$

(i) If $M-r<d$, then $\phi_{r}=0$.

(ii) If $0 \leqslant d \leqslant M-r$, then there is a non trivial homogeneous polynomial $\phi_{r}$ satisfying $(4)$.

(iii) If $d=M-r$ in particular, $\phi_{r}$ is equal to a constant multiple of $v=v_{A_{r}^{+}, m, \mathfrak{c}_{\text {nice }}}(a)$. 
Proof. We argue by induction on $r$. In the case that $r=1$, we write

$$
\phi_{1}=\phi\left(a_{1}\right)=p a_{1}^{d},
$$

where $p$ is a constant. If $m_{1,2}-1<d$ and $\phi_{1}$ satisfies the differential equation $\partial_{1}^{m_{1,2}} \phi_{1}=0$, then $p=0$ and hence $\phi_{1}=0$. If $0 \leqslant d \leqslant m_{1,2}-1$, then for any $p \neq 0, \partial_{1}^{m_{1,2}} \phi_{1}=0$. Also, if $d=m_{1,2}-1$, in particular, then $\phi_{1}=p a_{1}^{m_{1,2}-1}$, while $v=\frac{1}{\left(m_{1,2}-1\right) !} a_{1}^{m_{1,2}-1}$ as in Example 12. Hence $\phi_{1}$ is equal to a constant multiple of $v$.

We assume that the statement of this theorem holds for $r-1$. We write $\phi_{r}$ as

$$
\phi_{r}=\phi\left(a_{1}, \ldots, a_{r}\right)=g_{d}\left(a_{2}, \ldots, a_{r}\right)+a_{1} g_{d-1}\left(a_{2}, \ldots, a_{r}\right)+\cdots+a_{1}^{d} g_{0}\left(a_{2}, \ldots, a_{r}\right),
$$

where $g_{k}$ is a homogeneous polynomial of $a_{2}, \ldots, a_{r}$ with degree $k$ for $k=0,1, \ldots, d$. Then for $k=0,1, \ldots, d, g_{k}$ satisfies the differential equations as follows:

$$
\left\{\begin{array}{l}
\partial_{r}^{m_{r, r+1}} g_{k}=0 \\
\left(\partial_{r-1}-\partial_{r}\right)^{m_{r-1, r}} \partial_{r-1}^{m_{r-1, r+1}} g_{k}=0 \\
\quad \vdots \\
\left(\partial_{2}-\partial_{3}\right)^{m_{2,3}}\left(\partial_{2}-\partial_{4}\right)^{m_{2,4}} \cdots\left(\partial_{2}-\partial_{r}\right)^{m_{2, r}} \partial_{2}^{m_{2, r+1}} g_{k}=0 .
\end{array}\right.
$$

We set $h=\left(\sum_{2 \leqslant i<j \leqslant r+1} m_{i, j}\right)-(r-1)$. From the inductive assumption, if $0 \leqslant k \leqslant h$, then $g_{k}$ is a homogeneous polynomial. On the other hand, if $h+1 \leqslant k \leqslant d$, then $g_{k}=0$, namely,

$$
g_{d}\left(a_{2}, \ldots, a_{r}\right)=g_{d-1}\left(a_{2}, \ldots, a_{r}\right)=\cdots=g_{h+1}\left(a_{2}, \ldots, a_{r}\right)=0 .
$$

(i) We consider the case of $M-r<d$. Let $M_{1}=\sum_{i=2}^{r+1} m_{1, i}$. Now we compare the coefficients of $a_{1}^{d-h-M_{1}+n}$ in $\left(\partial_{1}-\partial_{2}\right)^{m_{1,2}}\left(\partial_{1}-\partial_{3}\right)^{m_{1,3}} \cdots\left(\partial_{1}-\partial_{r}\right)^{m_{1, r}} \partial_{1}^{m_{1, r+1}} \phi_{r}$ for $n=0, \ldots, h$. For $q=1, \ldots, M_{1}-m_{1, r+1}$, we define

$$
\begin{aligned}
D_{q}= & \sum_{2 \leqslant i_{1} \leqslant r}\left(\begin{array}{c}
m_{1, i_{1}} \\
q
\end{array}\right) \partial_{i_{1}}^{q}+\cdots+\sum_{\substack{p_{1}+\cdots+p_{k}=q \\
2 \leqslant i_{1}<\cdots<i_{k} \leqslant r}}\left(\prod_{1 \leqslant l \leqslant k}\left(\begin{array}{c}
m_{1, i_{l}} \\
p_{l}
\end{array}\right)\right) \partial_{i_{1}}^{p_{1}} \partial_{i_{2}}^{p_{2}} \cdots \partial_{i_{k}}^{p_{k}} \\
& +\cdots+\sum_{2 \leqslant i_{1}<\cdots<i_{q} \leqslant r}\left(\prod_{1 \leqslant l \leqslant q}\left(\begin{array}{c}
m_{1, i_{l}} \\
1
\end{array}\right)\right) \partial_{i_{1}} \partial_{i_{2}} \cdots \partial_{i_{q}} .
\end{aligned}
$$

Then we have the following equation:

$$
\begin{aligned}
& \frac{(d-h+n) !}{\left(d-h-M_{1}+n\right) !} g_{h-n}\left(a_{2}, \ldots, a_{r}\right)-\frac{(d-h+n-1) !}{\left(d-h-M_{1}+n\right) !} D_{1} g_{h-n+1}\left(a_{2}, \ldots, a_{r}\right) \\
& +\cdots+(-1)^{j} \frac{(d-h+n-j) !}{\left(d-h-M_{1}+n\right) !} D_{j} g_{h-n+j}\left(a_{2}, \ldots, a_{r}\right) \\
& +\cdots+(-1)^{M_{1}-m_{1, r+1}} \frac{\left(d-h+n-\left(M_{1}-m_{1, r+1}\right)\right) !}{\left(d-h-M_{1}+n\right) !} D_{M_{1}-m_{1, r+1}} g_{h-n+M_{1, r}}\left(a_{2}, \ldots, a_{r}\right) \\
& =0 .
\end{aligned}
$$


When $n=0$, from (7) and (8), we have

$$
g_{h}\left(a_{2}, \ldots, a_{r}\right)=0
$$

When $n=1$, we have

$$
\frac{(d-h+1) !}{\left(d-h-M_{1}+1\right) !} g_{h-1}\left(a_{2}, \ldots, a_{r}\right)-\frac{(d-h) !}{\left(d-h-M_{1}+1\right) !} D_{1} g_{h}\left(a_{2}, \ldots, a_{r}\right)=0 .
$$

Thus we have

$$
g_{h-1}\left(a_{2}, \ldots, a_{r}\right)=0
$$

Similarly, we have

$$
g_{h-2}\left(a_{2}, \ldots, a_{r}\right)=g_{h-3}\left(a_{2}, \ldots, a_{r}\right)=\cdots=g_{0}\left(a_{2}, \ldots, a_{r}\right)=0
$$

and hence $\phi_{r}=0$.

(ii) We consider the case of $0 \leqslant d \leqslant M-r$. By the inductive assumption, there is a non trivial homogeneous polynomial $g_{h-n_{1}+i}$ satisfying (6) for $i=1, \ldots, n_{1}$, where $n_{1}=M-r-d+1$. We can take

$$
g_{h-n_{1}+i}\left(a_{2}, \ldots, a_{r}\right) \neq 0 .
$$

When $n=n_{1}$, from (7) and (8),

$$
\begin{aligned}
g_{h-n_{1}}\left(a_{2}, \ldots, a_{r}\right)= & \frac{\left(d-h+n_{1}-1\right) !}{\left(d-h+n_{1}\right) !} D_{1} g_{h-n_{1}+1}\left(a_{2}, \ldots, a_{r}\right) \\
& -\frac{\left(d-h+n_{1}-2\right) !}{\left(d-h+n_{1}\right) !} D_{2} g_{h-n_{1}+2}\left(a_{2}, \ldots, a_{r}\right) \\
& +\cdots+(-1)^{n_{1}-1} \frac{(d-h) !}{\left(d-h+n_{1}\right) !} D_{n_{1}} g_{h}\left(a_{2}, \ldots, a_{r}\right) .
\end{aligned}
$$

When $n=n_{1}+1$,

$$
\begin{aligned}
g_{h-\left(n_{1}+1\right)}\left(a_{2}, \ldots, a_{r}\right)= & \frac{\left(d-h+n_{1}\right) !}{\left(d-h+n_{1}+1\right) !} D_{1} g_{h-n_{1}}\left(a_{2}, \ldots, a_{r}\right) \\
& -\frac{\left(d-h+n_{1}-1\right) !}{\left(d-h+n_{1}+1\right) !} D_{2} g_{h-n_{1}+1}\left(a_{2}, \ldots, a_{r}\right) \\
& +\cdots+(-1)^{n_{1}} \frac{(d-h) !}{\left(d-h+n_{1}+1\right) !} D_{n_{1}+1} g_{h}\left(a_{2}, \ldots, a_{r}\right) .
\end{aligned}
$$

Similarly, for $n=n_{1}+2, \ldots, h$, we can express $g_{h-j}\left(a_{2}, \ldots, a_{r}\right)\left(j=n_{1}, n_{1}+1, \ldots, h\right)$ in terms of $g_{h-j+i}\left(a_{2}, \ldots, a_{r}\right)(i=1, \ldots, j)$ and their partial derivatives. Namely, we can express $\phi_{r}$ in terms of $g_{h-n_{1}+i}\left(a_{2}, \ldots, a_{r}\right)$ and their partial derivatives. It follows that $\phi_{r} \neq 0$ when $0 \leqslant d \leqslant M-r$.

(iii) If $d=M-r$ in particular, then $n_{1}=1$, and $g_{h-j}(j=1, \ldots, h)$ becomes the linear combination of $g_{h}$ and their partial derivatives. Therefore $\phi_{r}$ is uniquely determined by 
$g_{h}$. Moreover, from the inductive assumption, $g_{h}=C \cdot v_{A_{r-1}^{+}, m^{\prime}, \mathfrak{c}_{\text {nice }}^{\prime}}$, where $C$ is a constant, $m^{\prime}=\left(m_{i, j}\right)_{2 \leqslant i<j \leqslant r+1}$, and $\mathfrak{c}_{\text {nice }}^{\prime}$ is a nice chamber of $A_{r-1}^{+}$. Hence the solution of (5) is unique up to a constant multiple. On the other hand, by Theorem $15, v_{A_{r}^{+}, m, \mathfrak{c}_{\text {nice }}}$ satisfies the system of differential equations (5). Hence $\phi_{r}$ is equal to a constant multiple of $v_{A_{r}^{+}, m, \mathfrak{c}_{\text {nice }}}$.

Recall that in the proof of Theorem 18, we have defined the operator

$$
\begin{aligned}
D_{q}= & \sum_{2 \leqslant i_{1} \leqslant r}\left(\begin{array}{c}
m_{1, i_{1}} \\
q
\end{array}\right) \partial_{i_{1}}^{q}+\cdots+\sum_{\substack{p_{1}+\cdots+p_{k}=q \\
2 \leqslant i_{1}<\cdots<i_{k} \leqslant r}}\left(\prod_{1 \leqslant l \leqslant k}\left(\begin{array}{c}
m_{1, i_{l}} \\
p_{l}
\end{array}\right)\right) \partial_{i_{1}}^{p_{1}} \partial_{i_{2}}^{p_{2}} \cdots \partial_{i_{k}}^{p_{k}} \\
& +\cdots+\sum_{2 \leqslant i_{1}<\cdots<i_{q} \leqslant r}\left(\prod_{1 \leqslant l \leqslant q}\left(\begin{array}{c}
m_{1, i_{l}} \\
1
\end{array}\right)\right) \partial_{i_{1}} \partial_{i_{2}} \cdots \partial_{i_{q}}
\end{aligned}
$$

for $q=1, \ldots, M_{1}-m_{1, r+1}$.

Remark 19. Let $M_{1}=\sum_{i=2}^{r+1} m_{1, i}$. When $d=M-r$, from the proof of Theorem 18 (iii), $g_{h-j}(j=1, \ldots, h)$ is uniquely determined as follows:

$$
\left\{\begin{aligned}
g_{h-1} & =\frac{\left(M_{1}-1\right) !}{M_{1} !} D_{1} g_{h} \\
g_{h-2} & =\frac{\left(M_{1}-1\right) !}{\left(M_{1}+1\right) !}\left(D_{1}^{2}-D_{2}\right) g_{h} \\
g_{h-3} & =\frac{\left(M_{1}-1\right) !}{\left(M_{1}+2\right) !}\left(D_{1}^{3}-2 D_{1} D_{2}+D_{3}\right) g_{h} \\
\quad & \\
g_{0}= & \frac{\left(M_{1}-1\right) !}{(M-r) !}\left(D_{1}^{h}-(h-1) D_{1}^{h-2} D_{2}+\cdots+(-1)^{h-1} D_{h}\right) g_{h} .
\end{aligned}\right.
$$

Let $m^{\prime}=\left(m_{i, j}\right)_{2 \leqslant i<j \leqslant r+1}, \mathfrak{c}_{\text {nice }}^{\prime}$ a nice chamber of $A_{r-1}^{+}$and $a^{\prime}=\sum_{i=2}^{r} a_{i}\left(e_{i}-e_{r+1}\right) \in$

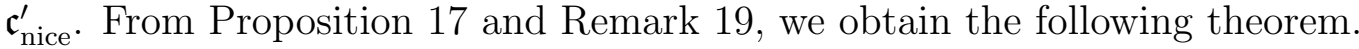

Theorem 20. Let $h=\left(\sum_{2 \leqslant i<j \leqslant r+1} m_{i, j}\right)-(r-1)$ and let $D_{q}(q=1, \ldots, h)$ be as in $(9)$. Then $v_{A_{r}^{+}, m, \mathfrak{c}_{\text {nice }}}(a)$ is written by the linear combination of $v_{A_{r-1}^{+}, m^{\prime}, \mathfrak{c}_{\text {nice }}^{\prime}}\left(a^{\prime}\right)$ and its partial derivatives as follows:

$$
\begin{aligned}
v_{A_{r}^{+}, m, \mathfrak{c}_{\text {nice }}}(a)= & \left\{\frac{a_{1}^{M_{1}-1}}{\left(M_{1}-1\right) !}+\frac{a_{1}^{M_{1}}}{M_{1} !} D_{1}+\frac{a_{1}^{M_{1}+1}}{\left(M_{1}+1\right) !}\left(D_{1}^{2}-D_{2}\right)\right. \\
& +\frac{a_{1}^{M_{1}+2}}{\left(M_{1}+2\right) !}\left(D_{1}^{3}-2 D_{1} D_{2}+D_{3}\right)+\cdots \\
& \left.+\frac{a_{1}^{M-r}}{(M-r) !}\left(D_{1}^{h}-(h-1) D_{1}^{h-2} D_{2}+\cdots+(-1)^{h-1} D_{h}\right)\right\} v_{A_{r-1}^{+}, m^{\prime}, \mathfrak{c}_{\text {nice }}^{\prime}}\left(a^{\prime}\right) .
\end{aligned}
$$

Example 21. Let $r=3$, let $a=\sum_{i=1}^{3} a_{i}\left(e_{i}-e_{4}\right) \in \overline{\mathfrak{c}_{\text {nice }}}$ and let $a^{\prime}=\sum_{i=2}^{3} a_{i}\left(e_{i}-e_{4}\right) \in \overline{\mathfrak{c}_{\text {nice }}^{\prime}}$. We set $m_{1,2}=1, m_{1,3}=1, m_{1,4}=2, m_{2,3}=1, m_{2,4}=2$ and $m_{3,4}=2$ as in Example 14 . Then we have

$$
v_{A_{3}^{+}, m, \mathfrak{c}_{\text {nice }}}(a)=\frac{1}{360} a_{1}^{3}\left(a_{1}^{3}+6 a_{1}^{2} a_{2}+3 a_{1}^{2} a_{3}+15 a_{1} a_{2}^{2}+15 a_{1} a_{2} a_{3}+10 a_{2}^{3}+30 a_{2}^{2} a_{3}\right) .
$$


We can check that $v=v_{A_{3}^{+}, m, \mathfrak{c}_{\text {nice }}}(a)$ satisfies the system of differential equations as follows:

$$
\left\{\begin{array}{l}
\partial_{3}^{2} v=0 \\
\left(\partial_{2}-\partial_{3}\right) \partial_{2}^{2} v=0 \\
\left(\partial_{1}-\partial_{2}\right)\left(\partial_{1}-\partial_{3}\right) \partial_{1}^{2} v=0
\end{array}\right.
$$

Also, from Proposition 17, the coefficient of the term $a_{1}^{3} a_{2}^{2} a_{3}$ is $\frac{1}{3 ! 2 ! 1 !}=\frac{1}{12}$. When $r=2$,

$$
v_{A_{2}^{+}, m^{\prime}, \mathfrak{c}_{\text {nice }}^{\prime}}\left(a^{\prime}\right)=\frac{1}{6} a_{2}^{2}\left(a_{2}+3 a_{3}\right) .
$$

Therefore, we have

$$
\begin{aligned}
& \left\{\frac{a_{1}^{3}}{6}+\frac{a_{1}^{4}}{24} D_{1}+\frac{a_{1}^{5}}{120}\left(D_{1}^{2}-D_{2}\right)+\frac{a_{1}^{6}}{720}\left(D_{1}^{3}-2 D_{1} D_{2}+D_{3}\right)\right\} v_{A_{2}^{+}, m^{\prime}, \mathfrak{c}_{\text {nice }}^{\prime}}\left(a^{\prime}\right) \\
& =\frac{a_{1}^{3} a_{2}^{3}}{36}+\frac{a_{1}^{3} a_{2}^{2} a_{3}}{12}+\frac{a_{1}^{4} a_{2}^{2}}{24}+\frac{a_{1}^{4} a_{2} a_{3}}{24}+\frac{a_{1}^{5} a_{2}}{60}+\frac{a_{1}^{5} a_{3}}{120}+\frac{a_{1}^{6}}{360}=v_{A_{3}^{+}, m, \mathfrak{c}_{\text {nice }}}(a)
\end{aligned}
$$

as in (10).

\section{Acknowledgements}

The third author was supported by JSPS KAKENHI Grant Numbers JP16K05137 and JP19K03475. The authors thank the referee for valuable comments.

\section{References}

[1] W. Baldoni, M. Beck, C. Cochet and M. Vergne. Volume computation for polytopes and partition functions for classical root systems. Discrete Comput. Geom., 35: 551$595,2006$.

[2] W. Baldoni-Silva and M. Vergne, Residues formulae for volumes and Ehrhart polynomials of convex polytopes. arXiv:math/0103097v1, 2001.

[3] W. Baldoni and M. Vergne. Kostant partitions functions and flow polytopes. Transform. Groups, 13(3-4): 447-469, 2008.

[4] M. Beck and S. Robins. Computing the Continuous Discretely, second edition. Undergraduate Texts in Mathematics, Springer, New York, 1997.

[5] C. Benedetti, R. S. González D’León, C. R. H. Hanusa, P. E. Harris, A. Khare, A. H. Morales and M. Yip. A combinatorial model for computing volumes of flow polytopes. Trans. Amer. Math. Soc., 372(5): 3369-3404, 2019.

[6] M. Brion and M. Vergne. Arrangements of hyperplanes I : Rational functions and Jeffrey-Kirwan residue. Ann. Sci. École. Norm. Sup., 32: 715-741, 1999.

[7] S. Corteel, J. S. Kim and K. Mészáros. Flow polytopes with Catalan volumes. C. $R$. Acad. Sci. Paris., 355(3): 248-259, 2017. 
[8] V. Guillemin, E. Lerman and S. Sternberg. Symplectic Fibrations and Multiplicity Diagrams. Cambridge University Press, New York, 1996.

[9] J. E. Humphreys. Introduction to Lie Algebras and Representation Theory. Graduate Texts in Mathematics, Springer, New York, 1972.

[10] L. C. Jeffrey and F. C. Kirwan. Localization for nonabelian group actions. Topology, 34: 291-327, 1995.

[11] B. V. Lidskii. Kostant function of the root system of $A_{n}$. Funct. Anal. Appl., 18: 65-67, 1984.

[12] K. Mészáros and A. H. Morales. Volumes and Ehrhart polynomials of flow polytopes. Math. Z., 293: 1369-1401, 2019.

[13] J. R. Schmidt and A. M. Bincer. The Kostant partition function for simple Lie algebras. J. Math. Phys., 25: 2367-2373, 1984. 